\title{
Macular hole treatment
}

\author{
Andrei Merticariu ${ }^{1}$, Florian Balta ${ }^{1,2}$, Mihai Pop ${ }^{1,2}$ \\ ${ }^{1}$ Clinical Emergency Eye Hospital, Bucharest, Romania \\ 2"Carol Davila" University of Medicine and Pharmacy, Bucharest, Romania
}

Corresponding author:

Andrei Merticariu, MD

Clinical Emergency Eye Hospital,

Bucharest, Romania

E-mail: andreimerticariu@yahoo.com

\section{ABSTRACT}

Objectives: In a period in which minimally invasive surgery revolutionises vitreo-retinal surgery, macular hole vitrectomy has benefited the most from this technical and technological progress. The objective of this paper is to review the modern surgical methods used for the treatment of macular holes and to detail the differences between the current surgical techniques.

Material and methods: Review all the published papers between 2000 and 2015 in PubMed and Medscape databases and also the recent specialty literature.

Results: We noticed a similarity between the lots studied and the research methods used, especially after the introduction of the Optical Coherence Tomography in the 1990's. There is still a significant difference between the surgical techniques studied, and none is yet considered "gold standard".

Conclusions: All the surgical methods currently used in the treatment for macular holes are highly effective, but all the small differences in the anatomical and functional results do not indicate a standardized method.

Key words: 25 Gauge vitrectomy, macular hole

\section{INTRODUCTION}

The macular hole $(\mathrm{MH})$ represents a total defect in all layers of the neurosenzorial retina at the foveolar level, in the central fundus and in the center of the visual field. The macular hole is an anatomical condition in which the treatment is mainly surgical and it remains a rare opthalmological pathology in the current clinical practice.

Macular holes are found in only 0.3 percent of the population, most frequently found in patients over 60 years old, predominantly females. The holes are produced at the foveolar level because of the reduced thickness of the retina at this level (around $225 \mu \mathrm{m}$ ) and the means through which the rupture of the retinal layers occurs can be explained by the centipede traction force exerted by the internal limiting membrane on the internal retinal layers, associated with anterior-posterior traction in cases with vitreous-macular adherence.

The non-surgical treatment methods studied so far (expectancy or minimally 
invasive) are taken into consideration only for the incipient stages of the disease (stages IA and IB) and have limited efficiency and utility. The expectative treatment is recommended in cases of stage I $\mathrm{MH}$, in which the expectancy of spontaneous closing is at approximately $50 \%$ of cases and where surgical treatment (vitrectomy) does not render a significant improvement of the visual acuity $(1,2)$.

The minimally invasive treatment consists of intravitreal injection of fibrinolitic agents (Microplasting, Ocriplasmin), aiming to determine the cleavage and detachment of the posterior hyaloid membrane from the surface of the retina and eliminating the viteo-foveal traction. This enzymatic vitreolysis is only useful in stage IIB to eliminate the retinal traction (with proven evidence of aprox. 50\%) and it does not have any effective result on a full macular hole. Thus, anatomical succes can be seen in around $40 \%$ of treated cases by inducing the spontaneous closing of the macular hole (1).

The majority of the existing articles related to this topic have similar methods, especially after the introduction of optical coherence tomography $(\mathrm{OCT})(3,4,5)$. The utility of OCT in the direct visualisation of the microscopic retinal structure, has made it become the gold standard paraclinical examination for diagnosing $\mathrm{MH}$. The OCT is also essential for staging, monitoring the evolution, assessing the anatomical post-operatory outcome and for the evaluation of the vitreo-retinal traction in the fellow eye.

Both fluorescein angiography and Visual Field examination render minimal information and they were rarely used in these studies.

\section{Classification}

Depending on the ophthalmoscopic and tomographic aspect, macular holes are classified in 4 stages: stage IA (incipient $\mathrm{MH}$; figure 1), stage IB (ocult $\mathrm{MH}$;

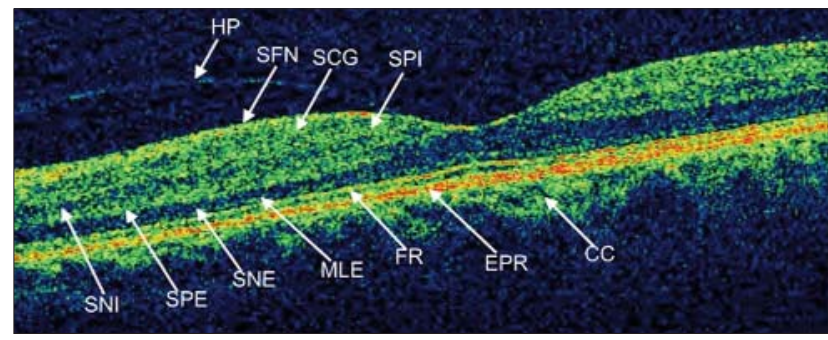

Figure 1 - OCT structure of normal retina

Legend: $\mathrm{HP}=$ posterior hyaloid; SFN = optic nerve fibers layer + internal limiting membrane; SCG = ganglion cell layer; $\mathbf{S P I}=$ inner plexiform layer; $\mathrm{SNI}=$ inner nuclear layer; $\mathrm{SPE}=$ outer plexiform layer; $\mathrm{SNE}=$ outer nuclear layer; MLE = external limiting membrane; SFR = layer of cones and rodes receptors; $E P R=$ pigmente depithelium; $C C=$ choriocapillaris figure 2), stage II (full-thickness $\mathrm{MH}$; figure 2), stage III (large dimensions $\mathrm{MH}$ with attached posterior hyaloid; figures 2,3) and stage IV (large dimensions $\mathrm{MH}$ with posterior vitreous detachment; figures 2,4,5).

The majority of studies published on this topic have used patients with advanced stages of MH (II-III-IV) with succesive surgical treatment, followed by a postoperative clinical and tomographic evaluation of minimum 6 - 12 months (5). The results have been analysed on two fronts: one is the percentage of anatomical closure of the macular hole and the other is the functional result (improvement of the visual acuity). The side effects and complications of the surgical techniques studied were similar.

\section{Treatment}

Pars plana vitrectomy (PPV) has always been the first choice treatment for macular holes and is the most used vitreoretinal surgical procedure nowadays. The vitrectomy is a complex surgical procedure which consists in the vitreous excision, frequently followed by other intraocular maneuvers. The procedure can be combined with other surgical techniques for the concomitant treatment of other ocular pathologies, such as phacoemulsification for cataract surgery (6).

The main surgical indication for vitrectomy in $\mathrm{MH}$ are stages II, III and IV $(2,3,7)$. Stage I macular holes close spontaneously in about $50 \%$ of cases and recent studies showed that surgery does not improve the outcome in these cases. Atrophic retinal holes without surrounding macular oedema or with a large diameter (usually in old or traumatic $\mathrm{MH}$ ) are relative contraindication for vitrectomy and in such cases the surgeon has to evaluate and estimate the procedure succes rate and to decide whether to perform or not the surgery. Macular holes with over six months from onset are also a relative contraindication for surgery, but several studies have shown that good results could also be achieved in patients with holes of over one year $(3,7)$.

PPV can be performed with either local or general anesthesia, depending on patient and surgeon preference. In recent years, vitreoretinal surgery has been routinely performed under local anesthesia due to the necessity forlong-lasting pain relief and akinesia, and also the important advantages of reduced procedure time, faster recovery and lower cost of admission (7).

Retrobulbar local anesthesia is the most commonly used due to its long-lasting effect and less frequent potential risks. General anesthesia with oral endotracheal intubation is still used for surgery on minor patients or with difficult collaboration $(3,7,8)$.

The basic surgical steps for pars plana vitrectomy in 


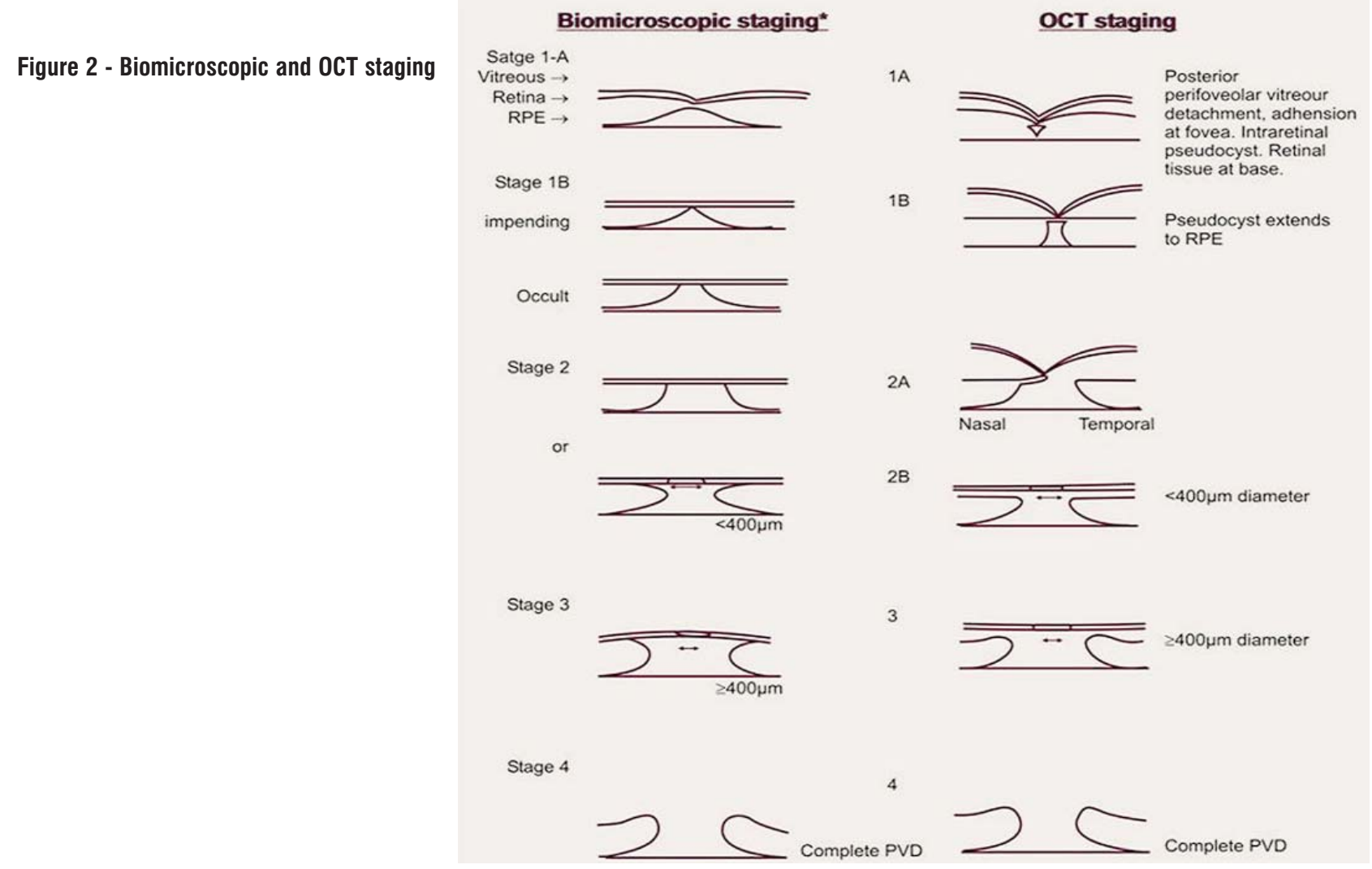

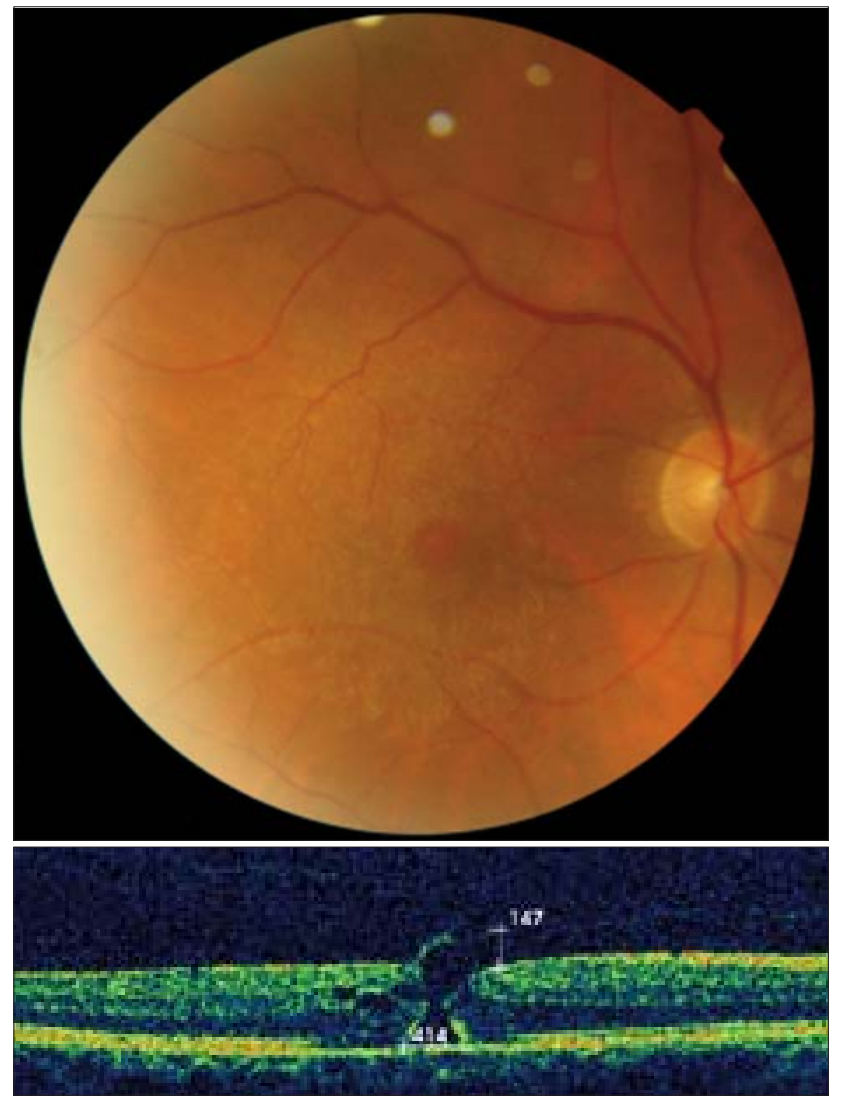

Figure 3 - clinical and OCT image of stage 3 macular hole

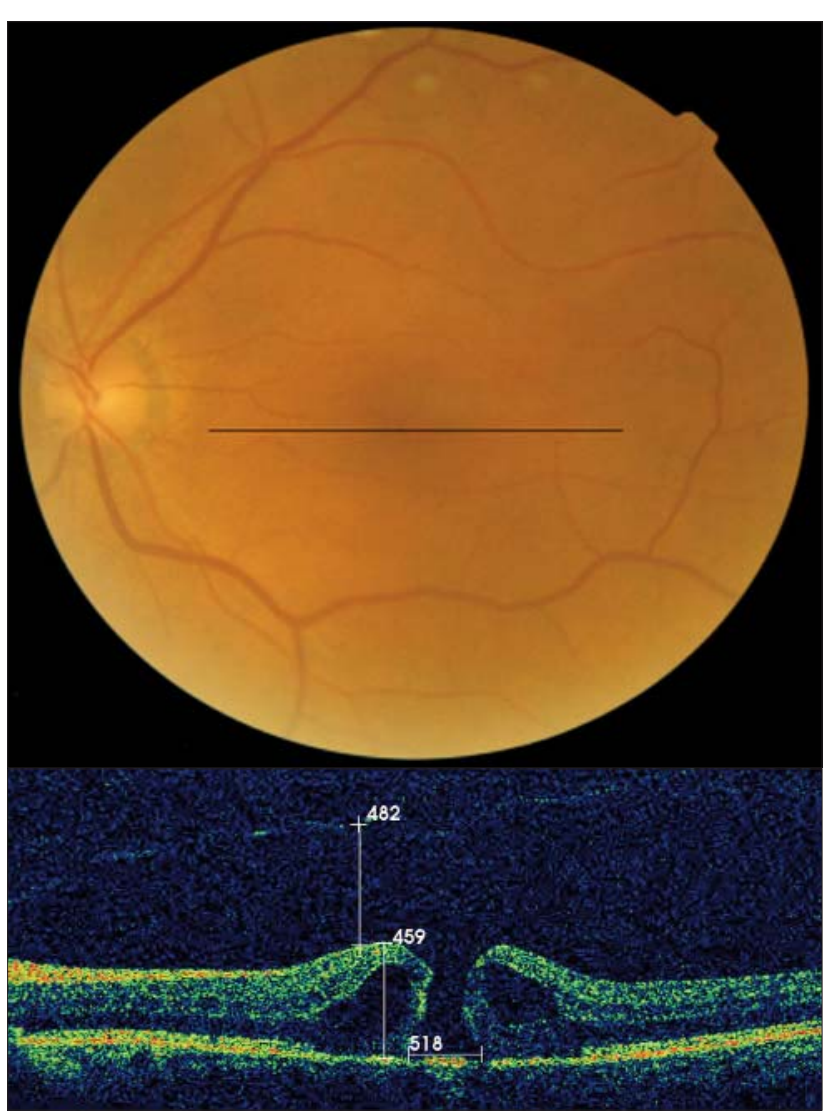

Figure 4 - preop clinical and OCT image of stage 4 macular 
$\mathrm{MH}$ cases, regardless of the technique used, consist in the full vitreous excision (as much as technically possible), detachment and excision of the posterior hyaloid, and retinal tamponade using intraocular air, expandable gasor silicone oil $(3,7,9)$. All the surgical techniques studied, published and used in the past 15 years meet this standard surgical protocol and the succes rate is analysed from the anatomical point-ofview (hole closure rate: figures 4, 5) and the functional improvement (increased visual acuity and central visual field scotoma remission).

Vitrectomy can be performed using 20 gauge $(0.813$ $\mathrm{mm}$ diameter), 23 gauge $(0.574 \mathrm{~mm}$ diameter)and 25 gauge $(0.455 \mathrm{~mm}$ diameter) instruments $(3,7,8) .2015$ is the year in which 27 gauge $(0.360 \mathrm{~mm}$ diameter) instruments became commercially available and 27 Gauge vitrectomy will probably be studied in the next few years. There are two ways of dealing with pars plana vitrectomy, and these are the classic 20 gauge method and the transconjunctival method using 23/25 gauge trocar systems $(10,11)$.

20 Gaugevitrectomy had been the gold standard since 1974, but after the year 2000 the transconjunctival suture less techniques became the most frequently studied and used in current practice (7). The $20 \mathrm{G}$ vitrectomy has the advantage of increased stiffness and

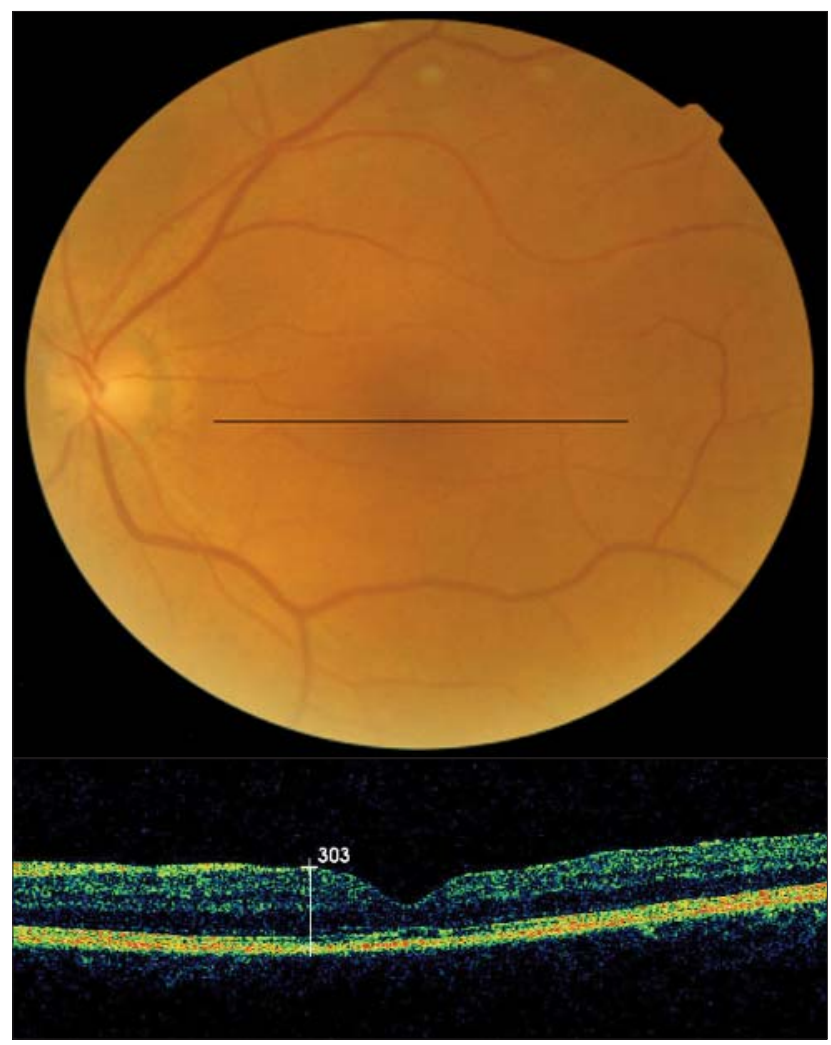

Figure 5 - postop clinical and OCT image of stage 4 macular hole strength of the instruments compared to those of 23/ $25 / 27 \mathrm{G}$, but the disadvantages are multiple and include incarceration of vitreous from the wound, secondary retinal break formation and the need to suture the sclera and the conjunctiva, which induces additional discomfort to the patient and increased expenses to the procedure. Thus, although the results of anatomical closure of the macular hole by the classical 20G technique are close to the percentage of modern techniques (over $60 \%$ ), the higher rate of complications and the increased medical and social costs have led the authors to focus their attention on the sutureless 23G/25G approach for the past 10 years $(10,11)$.

The advantages of the $25 \mathrm{G}$ transconjunctival vitrectomy include low surgical time and therefore a reduced need for long (general) anesthesia, lower rates of postoperative inflammation or infection, minimal conjunctival scarring, shorter recovery time, shorter hospitalization and procedure costs, superior comfort for the patient and faster visual recovery by sealing the eye without sutures $(3,10,11)$. Transconjuntival surgical treatment techniques for $\mathrm{MH}$ have a few significant differences from the classic method, regarding the use of intraocular dyes, the intraoperative hole closing, the ocular endotamponade and the postoperative patient positioning.

The internal limiting membrane (ILM) coloring and peeling (maculorhexis) is a frequently used surgical step, but it still has not become a standard procedure in MH surgery $(9,12,13)$. The main benefit from removing the ILM is that it will relieve the tangential tension on the inner retinal layers and improve the chances of hole closure. This is mostly the case in stage $4 \mathrm{MH}$, where there is no vitreo-retinal adherence and traction, and where only the centrifugal force is involved.

The dye originally used for staining and highlighting the internal limiting membrane (ILM) was Indocyanine green $(3,14,15)$ and, although it was of significant intraoperative help for the surgeon by shortening the intervention time and avoiding unwanted contact with the retinal layers, toxic side effects have imposed to limit its use (14), and to replace it with a less toxic coloring agent, respectively Trypan Blue or Brilliant Blue $(8,15)$. The most recent techniques have proposed eliminating the dye completely and to simply mark the ILM with Triamcinolone acetonide, an intraocular antiinflammatory corticosteroid commonly used in various eye disorders. This drug is used as a suspension containing white grains, which once injected in the eye, will deposited on the surface of the internal limiting membrane, thus marking it without any toxic effects (15). 
Intraoperative surgical $\mathrm{MH}$ closure is an optional maneuver and it consists of subretinal fluid aspiration through the hole after ILM peeling. The technique was described in multiple studies, both old and recent, and the debate is still ongoing (16). Some authors recommend avoiding it due to the extreme technical difficulty of this maneuver and the risk of touching and damaging the photoreceptors around the macular hole, which alters the functional outcome (17). The surgeons who have used this technique argue that the risk is justified because of the real-time intraoperative confirmation of the hole closure and because of proved higher anatomical closure rates. Another method recently studied involves coating large macular holes with an inverted ILM flappy reserved after the maculorhexis. The natural contractile tendency of the membrane due to glisosis will facilitate the edges to approach leading to the closure of the hole (18).

The final step of surgery consists of choosing the type of intraocular tamponade which can be either pneumatic retinopexy (air or expandable gases) or silicone oil. Pneumatic retinopexy uses expandable gas (usually C3F8 or SF6) and remains the first choice in $\mathrm{MH}$ surgical treatment. Once injected intravitrealy, expandable gases increase their volume and persist longer in the vitreal cavity, up to $4-6$ weeks $(9,19)$. If associated with long term postoperative head positioning, they provide a better retinal tamponade on the hole edges. However, expandable gases have a high risk of complications e.g. secondary glaucoma (increase in intra-ocular pressure) or proliferative vitreoretinopathy. The visual acuity is considerably reduced as long as the air or expandable gas persists in the vitreous cavity because it interferes with image focus on the retina. This is particularly important in single-eye patients, which will have a poor visual acuity up to six weeks postoperative.

Most studieson this topic confirm the ophthalmologists preference for gas tamponade in the last 15 years (19). Air tamponade is the most often technique used in vitreoretinal surgery for retinal detachment, vitreous haemorrhage, epiretinal membrane etc. However, air tamponade is not routinely used for surgical treatment of macular hole because it provides a weaker endotamponade. The air bubble resorbs over 5 to 7 days and it is gradually replaced with aqueous humour secreted by cilliary body, at which time the focus on the retina is restored and the visual acuity is restablished. Some authors consider that one week is enough to keep the macular hole closed and prefer the air tamponade because it has a better safety profile and a similar success rate as expandable gases or silicone oil but without their complications $(3,20)$. Moreover, air tamponade is significantly cheaper as surgical material, air being free, but also indirectly because of its rapid resorption which translates into fewer days of seek leave, better quality of life and better compliance when compared with expandable gas.

The silicone oil is less and less used in $\mathrm{MH}$ treatment and it is mainly reserved for recurrent cases nowadays (7). The main advantage of silicone oil consists of permanent and constant endotamponade. The silicone oil also allows to preserve an acceptable level of visual acuity although it changes the ocular refraction by +4 to +7 dioptres. Its disadvantages consist of neccesity for another surgical procedure to extract the oil after an average interval of three months and multiple side effects, especially the high risk of secondary glaucoma induced by emulsified silicone oil in the trabecular meshwork. In the current practice, low density silicone oil is used to minimize these risks, e.g. 1000,1300 or 2000 CentiStokes (21).

Postoperative patient positioning should be dorsally (face down), but the period of time needed is still debated $(9,20)$. This is important as it allows the efficient gas/oil bubble tamponade on the macula and it prevents the aqueous humour to reach the macular hole where it could potentially re-open it. Most authors recommend face down positioning for 7-10 days in order to maximise the intraocular tamponade force (3), although more recent studies show that there is no statistically significant diference when $\mathbf{4 8}$ hours or even 24 hours positioning was advised (20). Seven days positioning also implies significant higher medical and social costs, poorer compliance and major patient discomfort. Se recent studies analysed the posibility of avoiding dorsal postoperative positioning and the good outcomes suggest that positioning is less important as long as the surgical technique is very good $(19,22)$. However, these results need to be confirmed by future similar studies.

Although the success rates for 25 Gauge transconjunctival vitrectomy and expandable gas tamponade is usually around $90 \%$, the functional visual acuity noted in about $80 \%$ of cases $(3,7,23,24)$. The anatomical efficiency of 20 Gauge vitrectomy with silicone oil tamponade has been reported at around $65-80 \%$, but the functional success rates in these cases is much lower $(25,26)$. Most recent clinical studies showed that the rate of success of first intervention when using transconjunctival vitrectomy with air tamponade and short postop positioning may excede $90 \%$ of cases, without the multiple complications and higher procedure expenses of the previous techniques $(3,23,24)$. 


\section{CONCLUSIONS}

The use of new instruments, the refining of techniques and the better understanding of vitreoretinal physiopathology have led to consistent improvement of anatomical and functional outcomes following vitrectomy for $\mathrm{MH}$.

There is still a wide variety of surgery techniques used and most of them are highly successful. The small differences are still debated and, in the end, the vitreoretinal surgeon is the last to consider these nuances in the case at hand.

\section{Conflict of interests}

There is no conflict of interests

\section{Acknowledgement}

This paper is financially supported by the project "CERO - PROFIL DE CARIERĂ: CERCETĂTOR ROMÂN", under the contract number POSDRU/ 159/ 1.5/ S / 135760, project co-financed from the European Social Fund by the Sectoral Operational Programme "Human Resources Development 2007-2013".

\section{REFERENCES}

1. Stalmans P, Benz MS, Gandorfer A, Kampik A, Girach A, Pakola S, et al. Enzymatic vitreolysis with ocriplasmin for vitreomacular traction and macular holes. N Engl J Med. 2012 Aug 16;367(7):606-15.

2. Ullrich S, Haritoglou C, Gass C, Schaumberger M, Ulbig MW, Kampik A. Macular hole size as a prognostic factor in macular hole surgery. $\mathrm{Br}$ Ophthalmol. 2002 Apr;86(4):390-3.

3. Rizzo S, Belting C, Cresti F, Genovesi-Ebert F. Sutureless 25-gauge vitrectomy for idiopathic macular hole repair. Graefes Arch Clin Exp Ophthalmol. 2007 0ct;245(10):1437-40. Epub 2007 Mar 15.

4. Talu, S Balta, F Merticariu, A. Optical Coherence Tomography in Diagnosing and Monitoring of Retinal Diseases, Internationa Conference on advancements of Medicine and Health care through technology: 2009,26: 261-266.

5. Ip MS, Baker BJ, Duker JS, Reichel E, Baumal CR, Gangnon R, et al. Anatomical outcomes of surgery for idiopathic macular hole as determined by optical coherence tomography. Arch Ophthalmol. 2002 Jan;120(1):2935.

6. Theocharis IP, Alexandridou A, Gili NJ, Tomic Z. Combined phacoemulsification and pars plana vitrectomy for macular hole treatment. Acta Ophthalmol Scand. 2005 Apr;83(2):172-5.

7. Margherio A.R. Macular hole surgery in 2000. Curr Opin Ophthalmol. 2000 Jun:11(3):186-90.

8. Sanisoglu H, Sevim MS, Aktas B, Sevim S, Nohutcu A. Outcomes of 23- gauge pars plana vitrectomy and internal limiting membrane peeling with brilliant blue in macular hole. Clin Ophthalmol. 2011;5:1177-83.

9. Tadayoni R1, Gaudric A, Haouchine B, Massin P. Relationship between macular hole size and the potential benefit of internal limiting membrane peeling. Br J Ophthalmol. 2006 Oct;90(10):1239-41. Epub 2006 Jun 29

10. Kovacevic D, Antic IV, Valkovic A. Comparison of 23 gauge and 25 gauge PPV in the treatment of epiretinal membranes and macular holes. Coll Antropol. 2014 Dec;38(4):1213-6.

11. Kobayashi W, Kunikata H, Abe T, Nakazawa T. Retrospective Comparison of 25- and 23-Gauge Microincision Vitrectomy Surgery and 20-Gauge Vitrectomy for the Repair of Macular Hole Retinal Detachment. Asia Pac J Ophthalmol (Phila). 2014 Nov-Dec;3(6):331-6.

12. Lois N, Burr J, Norrie J, Vale L, Cook J, McDonald A, et al. Internal limiting membrane peeling versus no peeling for idiopathic full-thickness macular hole: a pragmatic randomized controlled trial. Invest Ophthalmol Vis Sci. 2011 Mar 1;52(3):1586-92.

13. Christensen, U. Value of internal limiting membrane peeling in surgery for idiopathic macular hole and the correlation between function and retinal morphology. Acta Ophthalmol. 2009 Dec;87 Thesis 2:1-23.

14. Wu Y, Zhu W, Xu D, Li YH, Ba J, Zhang XL, et al. Indocyanine greenassisted internal limiting membrane peeling in macular hole surgery: a meta-analysis. PLoS One. 2012;7(11):e48405.

15. Mochizuki N, Yamamoto T, Enaida H, Ishibashi T, Yamashita H. Long-term outcomes of 3 surgical adjuvants used for internal limiting membrane peeling in idiopathic macular hole surgery. Jpn J Ophthalmol. 2014 Nov; 58(6):455-61.

16. Shimada H, Hattori T, Nakashizuka H, Mori R, Mizutani Y, Yuzawa M. Highly viscous fluid in macular holes. Int Ophthalmol. 2010 Jun;30(3):319-21.

17. Tadayoni R, Svorenova I, Erginay A, Gaudric A, Massin P. Decreased retinal sensitivity after internal limiting membrane peeling for macular hole surgery. Br J Ophthalmol. 2012 Dec;96(12):1513-6.

18. Mahalingam, P. Sambhav, K. Surgical outcomes of inverted internal limiting membrane flap technique for large macular hole. Indian J Ophthalmol. 2013 Oct;61(10):601-3.

19. Rahman R1, Madgula I, Khan K. Outcomes of sulfur hexafluoride (SF6) versus perfluoroethane (C2F6) gas tamponade for non-posturing macularhole surgery. Br J Ophthalmol. 2012 Feb;96(2):185-8.

20. Tatham A. Banerje S. Face-down posturing after macular hole surgery: a meta-analysis. Br J Ophthalmol. 2010 May:94(5):626-31.

21. Karia N1, Laidlaw A, West J, Ezra E, Gregor MZ. Macular hole surgery using silicone oil tamponade. Br J Ophthalmol. 2001 Nov;85(11):1320-3.

22. Yagi, Fumihiko et al. Foveal microstructure and visual acuity in idiopathic macular holevitrectomy without postoperative face-down positioning. Folia Japonica de Ophthalmologica Clinica; 2011;4(9):888-92.

23. Veith M, Stranák Z, Pencák M, Studený P. Surgical Treatment of the Idiopathic Macular Hole by Means of 25-Gauge Pars Plana Vitrectomy with the Peeling of the Internal Limiting Membrane Assisted by Brilliant Blue and Gas Tamponade. Cesk Slov Oftalmol. 2015 Jun;71(3):170-4. [Article in Czech]

24. Carvounis PE1, Kopel AC, Kuhl DP, Heffez J, Pepple K, Holz ER. 25gaugevitrectomy using sulfur hexafluoride and no prone positioning for repair of macular holes. Retina. 2008 0ct;28(9):1188-92.

25. Lai JC, Stinnett SS, McCuen BW II. Comparison of silicone oil versus gas tamponade in the treatment of idiopathic full-thickness macular hole. Ophthalmology. 2003 Jun;110(6):1170-4

26. Karia N, Laidlaw A, West J, Ezra E, Gregor MZ. Macular hole surgery using silicone oil tamponade. Br J Ophthalmol. 2001 Nov;85(11):1320-3. 